\title{
NOTICE OF CONCERN
}

\section{Re: Thakur A, Kadam RS, and Kompella UB (2011) Influence of Drug Solubility and Lipophilicity on Transscleral Retinal Delivery of Six Corticosteroids. Drug Metab Dispos 39:771-781; doi:10.1124/dmd.110.037408}

\begin{abstract}
Drug Metabolism and Disposition is publishing this Editorial Notice of Concern regarding the integrity of the LC-MS/MS data in this article. An investigation by the University of Colorado determined that some LC-MS data in several other papers were falsified or fabricated by R. S. Kadam, who was the only respondent in the investigation. The Investigation Committee was unable to validate the LC-MS data in the above-referenced publication and thus could make no definitive determination relating to the integrity of the data in this manuscript.

Dr. Edward T. Morgan

Editor
\end{abstract}

